\title{
Influence de la nature de l'anion et du pH sur la conductivité hydraulique et la rétention en eau des pâtes de kaolinite sodique
}

\author{
Y. Daoud ${ }^{1}$ et M. Robert ${ }^{2}$ \\ 1 INA, Département de science du sol, Alger, Algérie; \\ 2 INRA, Station de science du sol, route de St-Cyr, 78000 Versailles, France
}

(reçu le 25 mai 1988, accepté le 26 mars 1989)

Résumé - La nature de l'anion présent dans la solution et le $\mathrm{pH}$ jouent un rôle important dans la conductivité hydraulique et la rétention en eau des pâtes d'une kaolinite sodique. En effet, les résultats obtenus montrent que la conductivité hydraulique et la rétention en eau à 0,1 bar diminuent dans le sens $\mathrm{Cl}>\mathrm{SO}_{4}>\mathrm{H}_{2} \mathrm{PO}_{4}$. En ce qui concerne le pH, les valeurs obtenues de ces 2 paramètres physiques diminuent dans le sens $\mathrm{pH} 4,20>\mathrm{pH} 5,40$. Ces résultats découlent des différences dans la micro-organisation des pâtes de kaolinite, et la microscopie électronique à balayage permet d'en visualiser certains aspects.

anions - sulfate - pH - conductivité hydraulique - rétention en eau - kaolinite sodique

Summary - Influence of the nature of the anion and $\mathrm{pH}$ on hydraulic conductivity and moisture retention in sodium kaolinite paste. The nature of the anion in solution and the $\mathrm{pH}$ play a major role in hydraulic conductivity and moisture retention of sodium kaolinite paste. The results obtained show that these two physical properties at 0,1 bar decrease with the nature of the anion in the following order $\mathrm{Cl}>\mathrm{SO}_{4}>\mathrm{H}_{2} \mathrm{PO}_{4}$ and with the $\mathrm{pH}: \mathrm{pH} 4.20>\mathrm{pH} 5.40$. These differences are related to the differences in microorganization of kaolinite pastes, some aspects of which may be clearly observed under scanning electron microscope.

anions - sulfate - pH - hydraulic conductivity - moisture retention - sodium kaolinite

\section{Introduction}

L'influence des cations échangeables sur les propriétés physiques des matériaux argileux a fait l'objet de nombreux travaux (Quirk et Schofield, 1955; Longenecker, 1959; McNeal et al., 1966; Hingston et al., 1972; Van Raij et Peech, 1972; El Swaify, 1973; Gallez et al., 1976; Prost, 1979; Halitim et al., 1984; Teissier et Berrier, 1979). En revanche, l'influence des anions sur les propriétés physiques des matériaux argileux est encore peu étudiée. Cette influence des anions est surtout importante pour les matériaux développant des charges de surface variables avec le $\mathrm{pH} d u$ milieu et la force ionique de la solution.

Par ailleurs, l'adsorption des anions varie avec la nature des colloïdes et le pH de la solution; c'est ainsi que pour une kaolinite et pour des valeurs de $\mathrm{pH}$ inférieures à 7 , les quantités d'anions adsorbées diminuent dans le sens $\mathrm{PO}_{4}$ $>\mathrm{SO}_{4}>\mathrm{Cl}$ (Bear, 1965).

Certains travaux indiquent que les anions peuvent exercer une influence importante sur les propriétés physiques des matériaux kaolinitiques. Les travaux de Johnson et Norton (1941), portant sur une kaolinite pure, montrent une différence considérable entre les effets des anions chlorure et sulfate sur la viscosité des suspensions de kaolinite. Les caractéristiques de charge de surface déterminent la stabilité des colloïdes et leur état de floculation. El Swaify (1973) a montré que la dispersion de la kaolinite sodique en équilibre avec une solution de $\mathrm{NaCl} 0,001 \mathrm{M}$ est maximale à partir de pH 8. Tama et EI Swaify (1977) situent le domaine de stabilité d'un matériau kaolinitique, en équilibre avec $\mathrm{KCl} 0,001 \mathrm{M}$, à un $\mathrm{pH}_{\mathrm{ZPC}}$ de 2,4 
$\left(\mathrm{pH}_{\mathrm{ZPC}}=\mathrm{pH}\right.$ au point de charge nulle). Ils montrent que la stabilité de ce matériau est atteinte quand la densité de charges positives de surface est de 0,86 10-7 meq. $\mathrm{cm}^{-2}$.

Le but de ce travail est de contribuer à l'étude de l'effet des anions chlorure, phosphate et sulfate sur la conductivité hydraulique et la rétention en eau des pâtes de kaolinite sodique. Ces mesures constituent un moyen d'évaluer l'effet de ces anions sur l'organisation du matériau étudié (Low, 1979; Prost, 1984).

\section{Matériel et Méthodes}

\section{Préparation de l'échantillon}

Pour ce travail, la fraction $<2 \mu \mathrm{m}$ du kaolin de Géorgie a été utilisée. Cette fraction a été extraite par sédimentation de la suspension à $\mathrm{pH} 9$ et débarrassée de l'excès d'ions $\mathrm{Na}^{+}$par lavage à l'éthanol à $60 \%$. L'argile obtenue est divisée en plusieurs lots. Chaque lot est lavé plusieurs fois avec l'une des solutions salines suivantes à $0,1 \mathrm{~N}: \mathrm{NaCl}$ à $\mathrm{pH} 4,20, \mathrm{NaCl}$ à $\mathrm{pH} 5,40$, $\mathrm{Na}_{2} \mathrm{SO}_{4}$ à pH 4,20, $\mathrm{Na}_{2} \mathrm{SO}_{4}$ à $\mathrm{pH} 5,40, \mathrm{Na}_{2} \mathrm{HPO}_{4}$ à $\mathrm{pH}$ $4,20, \mathrm{Na}_{2} \mathrm{HPO}_{4}$ à $\mathrm{pH} 5,40$. On considère que l'équilibre est atteint quand la concentration en anions de la solution d'équilibre reste constante et égale à celle de la solution utilisée, soit 100 meq.l-1. L'excès de sels est éliminé par une série de lavages avec la même solution à $0,001 \mathrm{~N}$.

\section{Techniques de mesure}

Les mesures de la conductivité hydraulique et de la rétention en eau

Elles ont été réalisées à l'aide d'un dispositif conçu par Prost (1984). Les solutions de percolation utilisées sont celles ayant servi aux lavages des échantillons (solutions à $0,001 \mathrm{~N}$ ). Les mesures sont réalisées à 0,1 bar. Les mesures de volume apparent par la méthode au pétrole (Monnier et al., 1973) montrent que les échantillons sont saturés en eau. L'épaisseur des échantillons est calculée en utilisant une valeur $\gamma_{1}$ de 2,63 g. $\mathrm{cm}^{-3}$. Les teneurs en eau sont déterminées par séchage des échantillons à $105^{\circ} \mathrm{C}$. La méthode de calcul de la conductivité hydraulique utilisée est celle décrite par Prost (1984).

\section{Les mesures de dispersion}

La technique suivie est celle préconisée par VelascoMolina et al. (1971). Les échantillons traités avec les différentes solutions salines sont séchés à $105{ }^{\circ} \mathrm{C}$. Ensuite $1 \mathrm{~g}$ d'argile est introduit dans un tube de centrifugeuse, auquel sont ajoutés $20 \mathrm{ml}$ de l'une des solutions salines étudiées $\left(\mathrm{NaCl}, \mathrm{Na}_{2} \mathrm{SO}_{4}, \mathrm{Na}_{2} \mathrm{HPO}_{4}\right)$ à $0,001 \mathrm{~N}$. La suspension est agitée pendant $15 \mathrm{~min}$ et ensuite centrifugée à $1000 \mathrm{t} / \mathrm{min}$ pendant $5 \mathrm{~min}$. La quantité d'argile contenue dans le surnageant est déterminée par séchage à $105{ }^{\circ} \mathrm{C}$, l'expression des résultats en pourcentage de la masse d'argile sèche donne le taux de dispersion.

\section{Détermination du point de charge nulle $(z p c)$}

II est déterminé par titration potentiométrique des suspensions d'argile, préparées avec des solutions de $\mathrm{NaCl}$ de concentrations différentes (Van Raij et Peech, 1972).
Toutes les mesures ont été réalisées en 3 répétitions. Le coefficient de variation de la méthode de mesure de la conductivité hydraulique, calculé sur 8 répétitions, est de $8 \%$, les mesures de teneur en eau ont un coefficient de variation de $2 \%$.

\section{Résultats et Discussion}

Les valeurs de conductivité hydraulique des pâtes de kaolinite obtenues sont comprises entre 28,9 et $128,910^{-8} \mathrm{~cm} \cdot \mathrm{sec}^{-1}$ (Fig. 1). Ces valeurs varient en fonction de l'anion utilisé et du $\mathrm{pH}$ des solutions. Pour un même $\mathrm{pH}$, les valeurs de conductivité hydraulique diminuent dans le sens $\mathrm{Cl}>\mathrm{SO}_{4}>\mathrm{H}_{2} \mathrm{PO}_{4}$. Les différences enregistrées entre les différents traitements sont variables. En effet, les rapports de conductivité hydraulique des pâtes de kaolinite $\left(K_{a}\right)$ entre les traitements sont les suivants :

$$
\begin{array}{r}
\text {-à pH 5,40: } \mathrm{K}(\mathrm{Cl}) / \mathrm{K}\left(\mathrm{SO}_{4}\right)=1,06 \\
\text { et } \mathrm{K}\left(\mathrm{SO}_{4}\right) / \mathrm{K}\left(\mathrm{H}_{2} \mathrm{PO}_{4}\right)=2,20 ; \\
\text {-à pH 4,20:K(Cl) } / \mathrm{K}\left(\mathrm{SO}_{4}\right)=1,11 \\
\text { et } \mathrm{K}\left(\mathrm{SO}_{4}\right) / \mathrm{K}\left(\mathrm{H}_{2} \mathrm{PO}_{4}\right)=1,28 .
\end{array}
$$

Pour le traitement avec un même anion, $\mathrm{K}$ varie avec le $\mathrm{pH}$, la valeur $\mathrm{K}$ obtenue à $\mathrm{pH} 4,20$ est supérieure à celle obtenue à $\mathrm{pH} 5,40$. La différence entre les valeurs de $\mathrm{K}$ entre $\mathrm{pH} \mathrm{4,20}$ et $\mathrm{pH} 5,40$ varie selon la nature des anions utilisés. En effet, les rapports $\mathrm{K} \mathrm{pH} \mathrm{4,20/K} \mathrm{pH} \mathrm{5,40} \mathrm{sont}$ de 1,80 pour $\mathrm{SO}_{4}, 1,88$ pour $\mathrm{Cl}$ et 3,10 pour

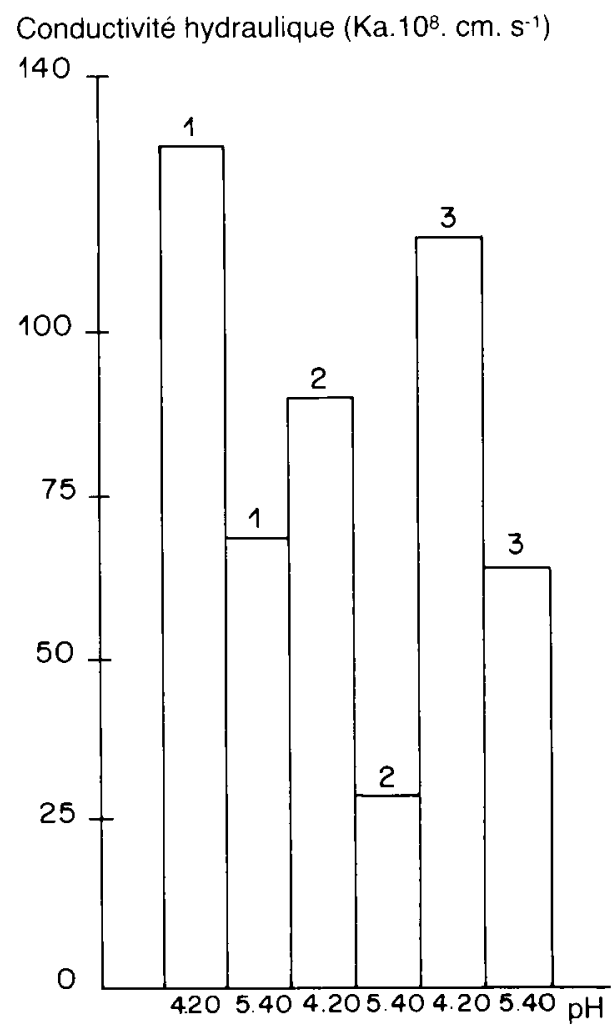

Fig. 1. Variation de la conductivité hydraulique en fonction des anions et du $\mathrm{pH}\left(1: \mathrm{Cl}, 2: \mathrm{H}_{2} \mathrm{PO}_{4}, 3: \mathrm{SO}_{4}\right)$. 
$\mathrm{H}_{2} \mathrm{PO}_{4}$. L'ensemble de ces résultats montre que les valeurs de conductivité hydraulique obtenues en fonction des anions et du $\mathrm{pH}$ des solutions diminuent dans le sens $\mathrm{Cl} 4,20>\mathrm{SO}_{4} 4,20>$ $\mathrm{H}_{2} \mathrm{PO}_{4} 4,20>\mathrm{Cl} 5,40>\mathrm{SO}_{4} 5,40>\mathrm{H}_{2} \mathrm{PO}_{4} 5,40$. Ces résultats révèlent donc que les anions utilisés ont une influence sur l'organisation des pâtes de kaolinite. En effet, les valeurs obtenues pour l'indice d'eau (teneur en eau en $\mathrm{cm}^{3}$ d'eau/ $\mathrm{cm}^{3}$ de solide) varient en fonction des anions et du pH (Fig. 2). La relation entre l'indice d'eau et le rayon hydraulique moyen, calculé selon la méthode décrite par Fernandez-Pineda et Mengual (1977), est linéaire $\left(r^{2}=0,85\right)$, elle montre que les pâtes de kaolinite se réorganisent différemment selon les traitements (Fig. 3). Plus généralement, les valeurs d'indice d'eau et de rayon hydraulique moyen varient dans le même sens que celles de la conductivité hydraulique; il faut cependant noter que ces 3 paramètres ne varient pas avec la même intensité, la variation

(Ө) $\left(\mathrm{cm}^{3}\right.$ d'eau $/ \mathrm{cm}^{3}$ de solide)

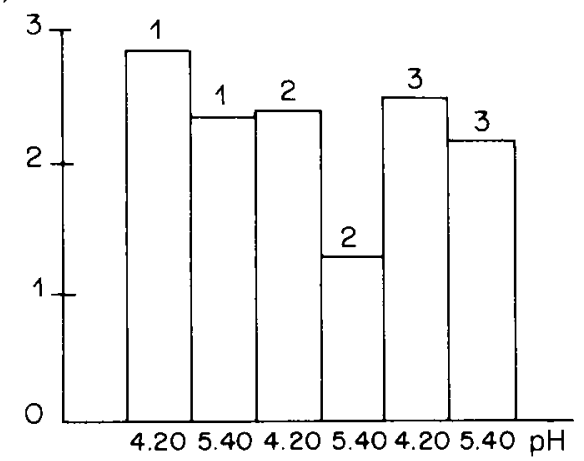

Fig. 2. Variation de l'indice d'eau en fonction des anions et du $\mathrm{pH}\left(1: \mathrm{Cl}, 2: \mathrm{H}_{2} \mathrm{PO}_{4}, 3: \mathrm{SO}_{4}\right)$.

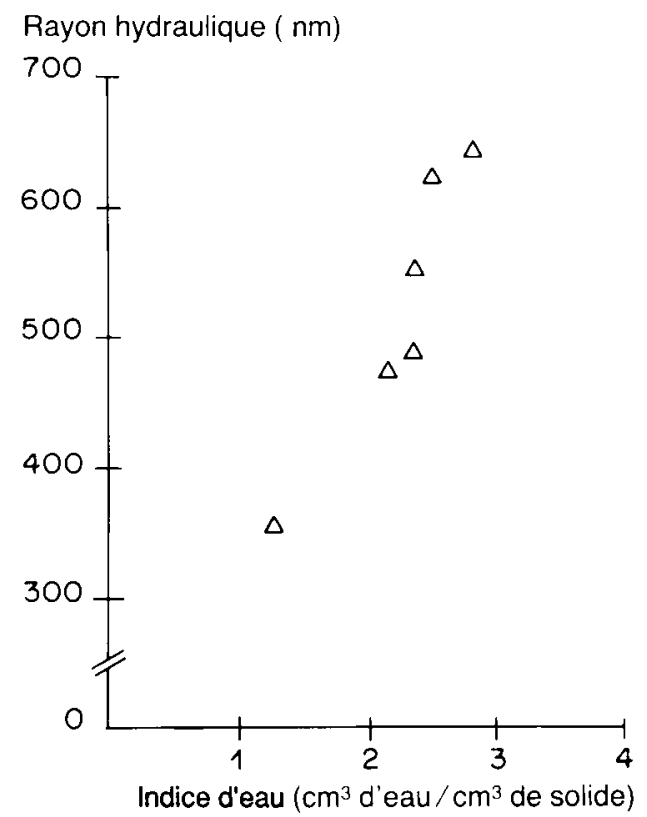

Fig. 3. Rayon hydraulique moyen des pâtes de kaolinite en fonction de l'indice d'eau. relative la plus élevée est observée pour la conductivité hydraulique.

Le ZPC déterminé dans des solutions de $\mathrm{NaCl}$ est obtenu à pH 3,20 pour cette kaolinite. Mais la détermination du ZPC peut être faussée si on utilise des anions qui se fixent sélectivement (Hingston et al., 1972). Par ailleurs, la balance des charges positives et négatives peut être modifiée par l'adsorption des phosphates, qui augmente la charge négative et donc le nombre de $\mathrm{H}^{+}$nécessaire en titration pour balancer cette charge (jusqu'à $0,80 \mathrm{meq} / 100 \mathrm{~g}$ de matériau kaolinitique à pH 7) (Mekaru et Uehara, 1972). La stabilité de ce type de matériau serait obtenue à des $\mathrm{pH}$ supérieurs de 1 à 3 unités au pH correspondant au ZPC (Tama et El Swaify, 1977). Dans notre

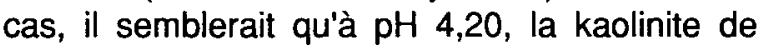
Géorgie soit à un $\mathrm{pH}$ correspondant au maximum de stabilité. En effet, les résultats montrent que le taux de dispersion augmente entre $\mathrm{pH} \mathrm{4,20} \mathrm{et}$ $\mathrm{pH} 5,40$, les taux de dispersion les plus élevés correspondant à l'anion phosphaté (Fig. 4). Les particules dispersées peuvent colmater certains pores et provoquer la diminution de la conductivité hydraulique du matériau argileux (Shainberg et al., 1981).

Par ailleurs, l'observation des pâtes de kaolinite séchées à 0,1 bar et préparées selon la technique décrite par Tessier et Berrier (1979) montre que l'organisation des particules de kaolinite diffère en fonction des traitements. Dans le cas des échantillons à pH 4,20, l'organisation des particules de l'échantillon- $\mathrm{SO}_{4}$ et de l'échantillon- $\mathrm{H}_{2} \mathrm{PO}_{4}$ est la suivante (Fig. 5) :

- échantillon $\mathrm{SO}_{4}$ : les particules ne sont pas orientées, leur arrangement est peu compact, la disposition des particules est en «château de

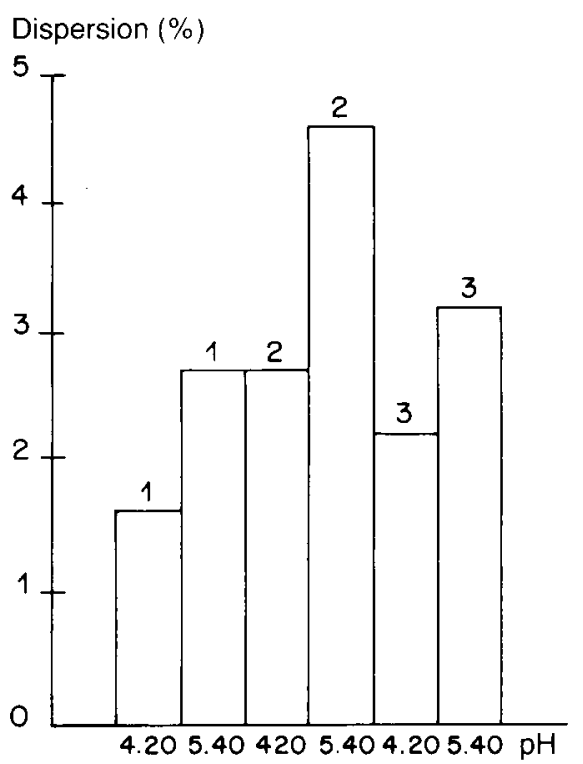

Fig. 4. Influence des anions et du pH sur le taux de dispersion $\left(1: \mathrm{Cl} 2: \mathrm{H}_{2} \mathrm{PO}_{4}, 3: \mathrm{SO}_{4}\right)$. 


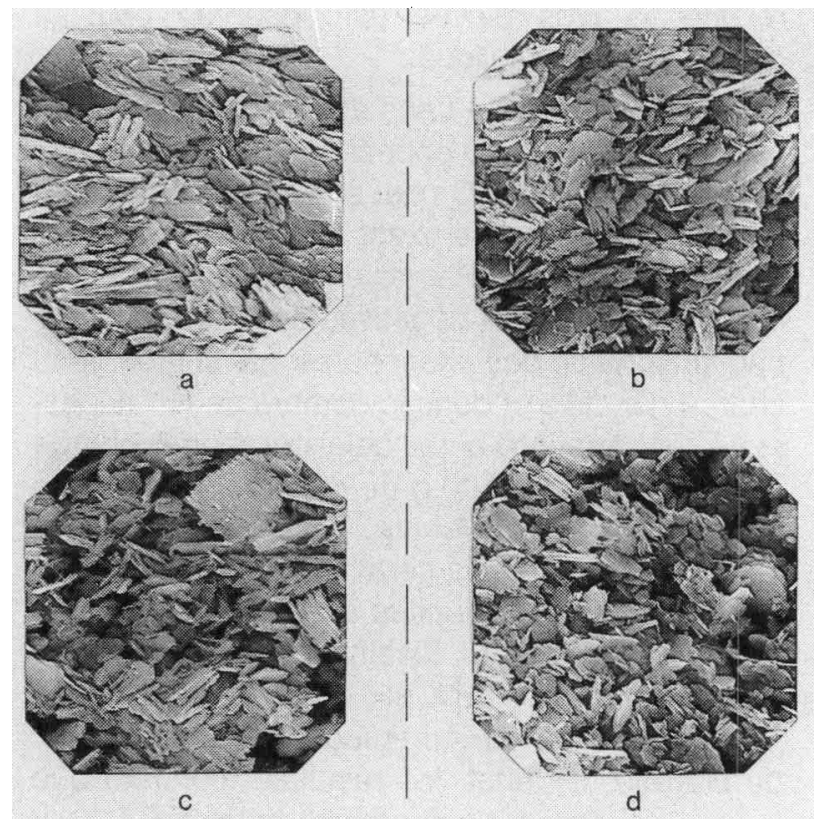

Fig. 5. Micro-organisation des pâtes de kaolinite à 0,1 bar (a et b : échantillon $\mathrm{H}_{2} \mathrm{PO}_{4}$ à pH 4,20; $\mathbf{c}$ et d : échantillon $\mathrm{SO}_{4}$ à pH 4,20). (photos J. Berrier, prises en microscopie à balayage avec dispositif cryoscan).

cartes", il existe de nombreux pores dans cet échantillon;

- échantillon $\mathrm{H}_{2} \mathrm{PO}_{4}$ : les particules ne sont pas orientées, leur arrangement est compact (liaisons des particules face à face), le nombre de pores est plus faible que celui observé sur l'échantillon précédent.

Pour l'effet du $\mathrm{pH}$, il semblerait que les différences de conductivité hydraulique obtenues entre $\mathrm{pH} 4,20$ et $\mathrm{pH} 5,40$ soient dues à une diminution du nombre de charges positives de bordure, qui permettent d'assurer un contact bord (à charges positives) - face (à charges négatives), créant ainsi un plus grand nombre de pores dans les pâtes de kaolinite (Van Olphen, 1963; Schofield et Samson, 1954).

\section{Conclusion}

Certaines propriétés physiques des pâtes de kaolinite sodique, telles que la conductivité hydraulique et la rétention en eau, semblent étroitement liées à la nature de l'anion compensateur et au pH du milieu. En effet, les principaux résultats obtenus montrent que la conductivité hydraulique et la rétention en eau diminuent dans le sens $\mathrm{Cl}>\mathrm{SO}_{4}>\mathrm{H}_{2} \mathrm{PO}_{4}$. Les observations au MEB montrent que la micro-organisation des pâtes de kaolinite diffère selon la nature de l'anion compensateur. La même série de manipulations prenant en compte l'effet $\mathrm{du} \mathrm{pH}$ montre que la conductivité hydraulique et la rétention en eau diminuent dans le sens $\mathrm{pH} 4,20>\mathrm{pH} \mathrm{5,40}$.
Ces résultats globaux mettent en évidence l'importance de la prise en compte de l'effet des anions et du $\mathrm{pH}$ sur le comportement physique des matériaux kaolinitiques : il semble cependant que les mécanismes sont encore insuffisamment élucidés, et des recherches s'imposent, en particulier sur les relations entre les variations de ZPC et les propriétés physiques des minéraux à charge variable.

\section{Références}

Bear F.E. (1965) Chemistry of The Soil. Theinold, New York

El Swaify S.A. (1973) Changes in the physical properties of soil clays due to precipitated aluminum and iron hydroxydes. II. Colloïdal interactions in the absence of drying. Soil Sci. 115, 64-72

Fernandes-Pineda C. \& Mengual J.I. (1977) Permeation of water of low pressure in cellulose acetate membranes. II. Nature of flow. J. Colloïde Interface Sci. 61, 95-108

Gallez A., Juo A.S.R. \& Herbillon A.J. (1976) Surface and changes caracteristics of selected soils in the tropics. Soil Sci. Soc. Am. J. 40, 601-608

Halitim A., Robert M. \& Prost R. (1984) Influence des cations échangeables $\left(\mathrm{Na}^{+}, \mathrm{Ca}^{++}, \mathrm{Mg}^{++}\right)$et de la concentration saline sur le comportement physique (rétention en eau, conductivité hydraulique) de la montmorillonite. Agronomie 4, 451-459

Hingston F., Posner A.M. \& Quirk J.P. (1972) Anions adsorption by geothite and gibbsite. I. The role of the proton in determining adsorption envelopes. J. Soil Sci. 23, 177-192

Johnson A.L. \& Norton F.H. (1941) Fundamental study of clay. J. Am. Ceram. Soc. 24, 189-203

Longenecker D.E. (1959) influence of soluble anions on some physico-chemical properties of soils. Soils Sci. 88, 185-191

Low P.F. (1979) Nature and properties of water in montmorillonite -water systems. Soil Sci. Soc. Am. J. 43, 651-658

McNeal B.L., Nowell W.A. \& Coleman N.T. (1966) Effect of solution composition on the swelling of extracted soils clays. Soil Sci. Soc. Am. Proc. 30, 313-317

Mekaru T. \& Uehara G. (1972) Anions adsorption in ferruginous tropical soils. Soil Sci. Soc. Am. Proc. 36, 296-300

Monnier G., Stengel P. \& Fies J.C. (1973) Une méthode de mesure de la densité apparente de petits agglomérats terreux. Application à l'analyse des systèmes de porosité du sol. Ann. Agron. 24, 533-545

Prost R. (1979) Application de mesures de conductivité hydraulique à l'étude de la structure des gels d'argile. Clay Miner. 14, 173-179

Prost R. (1984) Etude de la conductivité hydraulique des pâtes de kaolinite en fonction de l'entalpie libre $G$ du système argile-eau. Agronomie 4, 29-36

Quirk J.P. \& Schofield R.K. (1955) The effects of electrolyte concentration on soil permeability. J. Soil Sci. 6 , 163-178

Schofield R.K. \& Samson H.R. (1954) Floculation of kaolinite due to attraction of oppositely charged crystal faces. Discuss. Faraday Soc. 18, 135-145 
Shainberg I., Rhoades J.D. \& Prather R.J. (1981) Effect of low electrolyte concentration on clay dispersion and hydraulic conductivity of a sodic soil. Soil Sci. Soc. Am. J. 45, 273-277

Tama K. \& El Swaify S.A. (1977) Charge Colloïdal and Structural Stability Interrelationships for Oxidic Soils. Interscience Publishers, New York

Tessier D. \& Berrier J. (1979) Utilisation de la microscopie électronique à balayage dans l'étude des sols. Observations des sols humides soumis à différents pF.
Bull. Assoc. Jr. Etude Sol 1, 67-82

Van Olphen H. (1963) An Introduction to Clay Colloïd Chemistry. Interscience Publishers, New York

Van Raij B. \& Peech M. (1972) Electrochemical properties of some oxisols and alfisols of the tropics. Soil Sci. Soc. Am. Proc. 36, 587-593

Velasco-Molina H.A., Swoboda A.R. \& Godfrey C.L. (1971) Dispersion of soils of different mineralogy in relation to sodium adsorption ratio and electrolyte concentration. Soil Sci. 5, 282-287 